with a mixture of ox-serum, glucose, and milk, with liquor pancreaticus. This, he says, contains a constant quantity of proteid, which is easily absorbed by the mucous membrane of the large intestine, is inexpensive, and does not require any tedious preparation-simply the addition of a preservative. He has been in the habit of adding two grains of chloretone to each ounce of serum, since that drug not only acts as an antiseptic, but also as a sedative. From analyses of the material removed by the water enema, which is given every day to wash out the rectum, it was found that less than a gramme of proteid was excreted unaltered at a time when 51 grammes per day were being administered by enemata repeated every four hours. Carbohydrates in the form of starch or glucose are readily absorbed when given in solution by the rectum, and if the strength of the solution is less than 15 per cent. it is well retained and not irritating; so that 90 grammes may be introduced during the twenty-four hours. Fat is not easily absorbed, and of the 18 grammes contained in the milk some has invariably been returned in the wash-out enemata. It would appear that where it is desired to introduce nutriment by the rectum, ox-serum is a useful substitute for other forms of food, and enables us to administer a considerably larger quantity of proteid than could otherwise be got in. There is another great advantage in ox-serum over egg albumin-namely, that it does not give rise to offensive stools.

$$
{ }^{1} \text { Brit. Med. Jour., A pril } 6 .
$$

\section{ARSENIC IN THE HAIR.}

WheN a person is taking arsenic he excretes arsenic, be the same more or less, so that it may be possible to ascertain whether or not arsenical poisoning is actually or has recently been going on by a chemical examination of the urine. It not infrequently, however, becomes a question, not so much whether a person is now being poisoned, as whether certain symptoms, peripheral neuritis, for example, are due to an exposure to arsenical poisoning which may have ceased for some time. As one means of solving this problem it has been shown by Mr. W. Kirkby that, from the predilections of arsenic for the skin, it has been possible to detect it in a scab from a patient's body, and thus to confirm a diagnosis of arsenical neuritis. All patients, however, do not have scabs or scales which can be analysed, but most patients have hair, and it has been shown by Dr. Knecht and Dr. Dearden, ${ }^{1}$ of Manchester, that it is possible by chemical examination of one gramme of hair, removed from a patient, to obtain the evidence required. In the hair of a patient who had been taking arsenic medicinally (one-ninth of a grain per diem) it was found in the proportion of 03 in 10,000 , and the same proportion was found in a patient suffering from well marked arsenical beer poisoning, while in another beer poisoning case arsenic was found in the hair in the proportion of 1 in 10,000. In control experiments made on the hair of healthy individuals, although arsenic was shown to be present in traces, it existed in quantities too minute to be estimated in one gramme of substance. Clearly this gives a hint which may be of service in elucidating the nature of obscure cases. All the same, its indications must be accepted with certain limitations, especially in medicolegal cases. Apparently arsenic once deposited in the hair would remain there; and the more we know of the many hitherto unsuspected modes in which arsenic may obtain access to articles of food, the less becomes the value as evidence of crime of any such proof as examination of the hair will offer of bygone arsenical infiltration.

Commenting on the above article, Dr. A. Dupré ${ }^{2}$ says that some years ago in experimenting on the diffusion of various substances into and out of the animal tissues, he made use of chloride of lithium, and found that when this salt was administered the metal lithium was present in the hair, as well as in other tissues of the body. With the object of finding out whether this was the result of (1) direct diffusion into the hair; (2) absorption from the perspiration of the skin of the scalp; or (3) absorption from the hand passed through the hair, he carefully isolated some locks of hair by wrapping them in waterproof material, so as to prevent absorption by either of the last two possible means. Lithium, however, was never found in the hair so protected, and he came to the conclusion therefore that lithium did not diffuse directly into the hair. The question then is what would be the result in regard to arsenic were the same precautions taken.

2 Lancet, March 23. ' Lancet, April 6.

\section{CACODYLIC ACID.}

WHILE on the question of arsenic it may be well to refer to some remarks made by $\mathrm{Mr}$. Hutchinson in this month's Polyclinic. Speaking of the claims made for cacodylic acid, to the effect, namely, that it may be administered in large doses without disagreement and that it does not, like other preparations of arsenic, cause discoloration of the skin or peripheral neuritis, he says that it may be the fact that we really want a drug which is capable of producing these very effects. What we want to know is not that a considerable dose of arsenic can be given, but that the disease will be cured. The good effects of cacodylate of sodium as vaunted are, he says, precisely those which we have hitherto attributed to the older preparations of arsenic, but we must not assume that because a poison can be given in larger quantities in one salt than it can in another, therefore the one best tolerated is the better remedy; it is possible that it may be simply the less efficient.

\section{INTRA-VENOUS SALINE INFUSION IN DELIRIUM TREMENS.}

Thaт delirium tremens is a form of toxæmia one can hardly doubt, and Dr. Warbasse, ${ }^{1}$ of New York, proposes to treat it, as other forms of toxæmia have been treated, by the intra-venous injection of saline solution; in other words by washing out the poison. He says that the disease is characterised by auto-intoxication. A chronic degeneration of the glandular structures of the body results from the prolonged use of alcohol, with the result that when the organism suffers from the shock of a traumatic injury the secreting and excreting structures fail, and a toxæmia results. The identity of the peculiar toxins of delirium tremens has not, however, as yet been determined, but, whatever they are, the disease results from the action on the nervous system of toxins, which by virtue of the pathological conditions present fail to become eliminated, absorbed, or neutralised. In the majority of the cases the natural resources of the organism are sufficient to overcome the toxæmia. Sometimes, however, the intoxication is fatal, even in the presence of kidneys which are able to excrete an adequate amount of urea. By saline injection one increases the amount of the circulating medium in which the toxic materials are dissolved, thereby diluting the 